\title{
La cromoendoscopia virtual i-Scan y su aplicación en la detección y caracterización de los pólipos de colon
}

\author{
Virtual Chromoendoscopy with I-Scan and its Application for Detection and \\ Characterization of Colon Polyps
}

Henry Royero G., MD. ${ }^{1}$

1 Internista Gastroenterólogo. Miembro Internacional,
ACP, AGA, ASGE. Hospital "Emiro Quintero
Cañizares". Ocaña. Colombia.
Fecha recibido: $\quad 05-05-16$
Fecha aceptado: $16-12-16$

Fecha aceptado: $16-12-16$

\begin{abstract}
Resumen
La limitación de la colonoscopia con luz blanca respecto a la omisión de neoplasias avanzadas en un 6\%, y de adenomas hasta un $25 \%$, ha motivado el desarrollo de técnicas como la cromoendoscopia virtual, entre ellas el sistema i-Scan, para detectar un mayor número de pólipos, como estrategia de prevención del cáncer de colon. Con esta revisión se resume la aplicación actual de este método en la detección de adenomas y su caracterización. Se ha encontrado que este nuevo sistema es una alternativa posible en nuestro medio a las ya existentes y mejor conocidas, como el NBI y FICE.

\section{Palabras clave}

i-Scan, colonoscopia, pólipo.

Abstract

The limitations of white light colonoscopy result in missing $6 \%$ of advanced neoplasias in $6 \%$ and up to $25 \%$ of adenomas. This has motivated the development of techniques such as virtual chromoendoscopy including the i-Scan system to detect larger numbers of polyps as a strategy for preventing colon cancer. This review summarizes the current application of this technique for detection and characterization of adenomas. The new system is a possible alternative in our environment to the better known NBI and FICE which are already being used here.
\end{abstract}

\section{Key words}

i-Scan, colonoscopy, polyp.

\section{INTRODUCCIÓN}

La polipectomía de adenomas detectados durante la colonoscopia ha logrado reducir la incidencia de cáncer colorrectal (1-3); no obstante, se ha encontrado que hasta un $6 \%$ de las neoplasias y un $25 \%$ de los pólipos podrían no ser observados $(4,5)$. Lo anterior dio paso a varias modalidades diagnósticas, como la cromoendoscopia con colorantes vitales, que detecta un mayor número de pólipos, además de caracterizarlos mediante la clasificación de Kudo (6-8); sin embargo, no se recomienda el uso rutinario de esta técnica por lo dispendioso de su aplicación y debido a que podría prolongar el tiempo de exploración. Estos inconvenientes permiten que las nuevas técnicas de cromoendoscopia virtual sean más atractivas (9), que tienen las ventajas de ser reversibles, rápidas, limpias y sin efectos adversos por los colorantes (10). Actualmente existen cinco programas de cromoendoscopia virtual: la banda angosta (NBI) 
(Olympus), el sistema de imagen de banda compuesta (CBI) (Ahuoa), el realce inteligente de color de Fujifilm (FICE) y el sistema i-Scan (Pentax) (11). Recientemente se introdujo el SPIES (Storz) (12).

Esta es la primera revisión de tema sobre el sistema i-Scan en nuestro medio, cuyos objetivos son conocer esta tecnología y dar un punto de vista adicional a la colonoscopia de alta definición y a la cromoendoscopia virtual. Se realizó una búsqueda en Pubmed hasta enero de 2016, con las palabras claves $i$-Scan y endoscopy; se encontraron 68 artículos. No se incluyeron los diseñados para otras aplicaciones diferentes a la detección y caracterización de neoplasias de colon. También se revisaron las referencias de los trabajos seleccionados, incluida la bibliografía más relevante.

\section{¿QUÉ ES EL SISTEMA I-SCAN?}

El i-Scan de alta definición, desarrollado por Pentax (HOYA, Japón), está basado en el procesamiento de la imagen captada por el CCD (charge coupled device) del endoscopio para aumentar el contraste de las estructuras vasculares y de los patrones de la mucosa del tracto digestivo $(13,14)$, con resoluciones ópticas hasta de 1300000 pixeles (15-18).

El i-Scan consiste en tres modos basados en varios algoritmos: realce de superficie (surface enhancement; SE), realce de contraste (contrast enhancement; CE) y realce de tono (tone enhancement; TE). Los dos primeros, a su vez, tienen tres niveles: bajo, medio y alto. Con el TE pueden obtenerse tres objetivos (esófago, estómago y colon). Los tres modos (SE, CE y TE) están ordenados en serie; por tanto, pueden aplicarse dos o más de estos en forma consecutiva y en tiempo real, sin mayores retrasos, tan solo pulsando un botón $(11,19-21)$.

\section{¿CÓMO USAR LA TECNOLOGÍA I-SCAN?}

Basado en las recomendaciones de diferentes autores (15, $21,22)$, los modos del i-Scan están indicados en los siguientes escenarios:

- i-Scan 1 (SE) para la detección de lesiones refina la imagen de sutiles anormalidades de superficie sin alteración en la brillantez de la imagen endoscópica.

- i-Scan 2 (TE más SE), establecido para la caracterización de lesiones combinando realce de superficie y tono y realzando cambios diminutos de la mucosa y estructuras vasculares.

- i-Scan 3 (TE más CE) sirve para demarcación de lesiones; digitalmente adiciona color azul y oscurece los bordes de la imagen endoscópica.

\section{APLICACIÓN DEL I-SCAN EN LA DETECCIÓN DE PÓLIPOS DEL COLON}

El primer estudio prospectivo y aleatorizado que mostró resultados en la detección de pólipos con la metodología i-Scan detectó significativamente más neoplasias colorrectales en relación con la endoscopia de resolución estándar con luz blanca ( $38 \%$ versus $13 \%$; $\mathrm{p}<0,001)$ (23). Otro estudio prospectivo y aleatorizado (24) comparó la detección de adenomas entre el i-Scan con realce de tono versus la colonoscopia de alta definición con luz blanca. El realce de tono encontró más adenomas ( 20 versus $6 ; \mathrm{p}=0,006)$ e identificó un mayor número de pólipos omitidos por sujeto $(0,5$ versus 0,$15 ; \mathrm{p}=0,006)$. Se concluyó que el $\mathrm{i}$-Scan con realce de tono redujo el número de adenomas omitidos con la luz blanca. Bowman (25) encontró hallazgos significativamente similares con un mayor número de pacientes.

Otro estudio prospectivo (26), que incluyó 389 pacientes asintomáticos, tuvo por objetivo principal determinar los porcentajes en la detección de adenomas. Basado en un análisis multivariado, concluyó que el i-Scan no pudo documentar un rendimiento significativo en la detección de adenomas omitidos, lo que controvierte a los estudios ya citados, aunque tuvo una tendencia a mejorar la detección de adenomas utilizando el i-Scan en los modos SE/ TE-CE comparado con la luz blanca de alta definición. Este estudio tiene como limitante que fue realizado en un solo centro y con pocos endoscopistas.

\section{CARACTERIZACIÓN DE PÓLIPOS MEDIANTE EL I-SCAN}

El grupo de Mainz, en Alemania, fue el primero en estudiar la detección y caracterización de pólipos diminutos menores de $5 \mathrm{~mm}$. Con respecto a la predicción histológica con la técnica i-Scan, demostraron que los adenomas pueden predecirse con una sensibilidad de $9 / 11(82 \%)$ y una especificidad de 52/54 (96\%) (27).

Otro estudio prospectivo pudo predecir el resultado histológico con alta seguridad $(96,85 \%)$ a través de la clasificación de Kudo (23). Sucesivos estudios con este objetivo primario $(16,28,29)$ o secundario $(30)$ han comprobado la alta precisión del i-Scan en la clasificación de los pólipos en adenomatosos y no adenomatosos.

Un metaanálisis (31) que incluyó NBI, i-Scan, FICE, endomicroscopia confocal e imagen con autofluorescencia encontró que el i-Scan tuvo una sensibilidad del 89,3\% $(83,3-93,3)$ y una especificidad del $88,2 \%(80,3-93,2)$ en la predicción de pólipos, con un valor predictivo negativo en tiempo real del $86,5 \%(78-92,1)$. Estos datos fueron 
similares a las otras modalidades, por lo que se concluyó que estos métodos, incluido el i-Scan, podrían utilizarse de forma rutinaria.

Al comparar el i-Scan con otra técnica de cromoendoscopia virtual, como el NBI, no se encontraron diferencias evidentes entre las dos para predecir en tiempo real los pólipos menores de $5 \mathrm{~mm}$ (sensibilidad del $88,8 \%$ versus del 94,6\%; especificidad del $86,8 \%$ versus del $86,4 \%$; seguridad del $87,8 \%$ versus del $90,7 \%$, respectivamente) (29). Por otra parte, para la caracterización de los pólipos, Bouwens (32) propuso una sencilla clasificación que brinda una seguridad del $84 \%$ (clasificación ICE) (tabla 1 y figuras 1 a 10). Adicionalmente, Lee (29) plantea una clasificación unificada para el NBI y el i-Scan, que combina el patrón vascular y la descripción de la superficie mucosa de un pólipo. Ambas clasificaciones constituyen herramientas prácticas para el diagnóstico óptico en tiempo real en la caracterización de los pólipos adenomatosos.

Tabla 1. Clasificación de pólipos no adenomatosos y adenomatosos utilizando el sistema i-Scan (clasificación ICE) (32)

\begin{tabular}{lll}
\hline & \multicolumn{1}{c}{ No adenomatoso } & \multicolumn{1}{c}{ Adenomatoso } \\
\hline Color & $\begin{array}{l}\text { Pálido } \\
\text { Similar a la mucosa } \\
\text { adyacente } \\
\text { Bordes indiscretos }\end{array}$ & $\begin{array}{l}\text { Rojizo } \\
\text { Diferente a la mucosa } \\
\text { adyacente } \\
\text { Bordes claramente } \\
\text { demarcados }\end{array}$ \\
$\begin{array}{lll}\text { Patrón de } \\
\text { superficie }\end{array}$ & Fosos redondos de tamaño \\
Patrón de & fosos ovales, tubulares o \\
vasos & Aislados, delicados, & ramificados \\
\hline
\end{tabular}

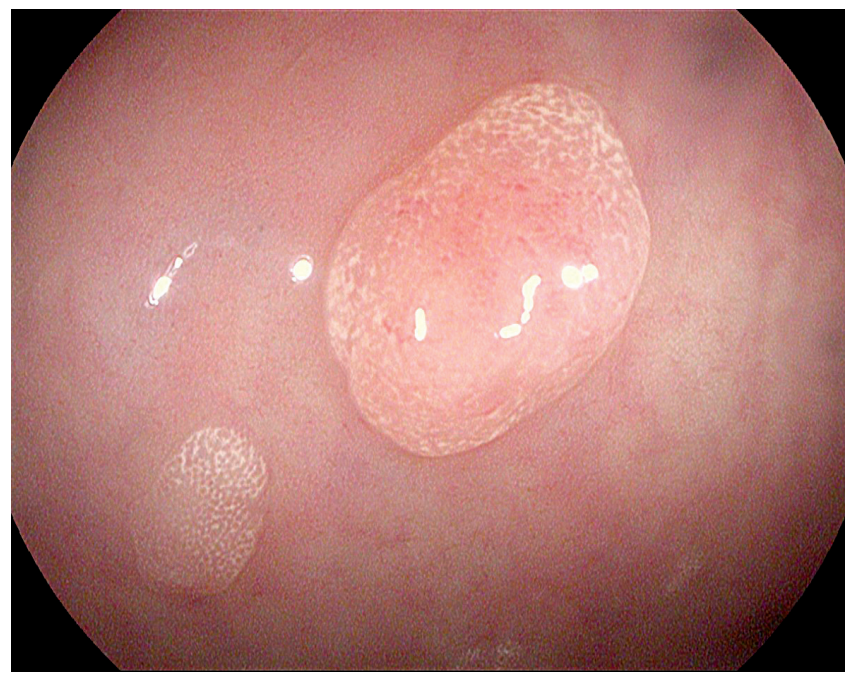

\section{Curva de aprendizaje en la caracterización de pólipos}

Según los hallazgos de un estudio prospectivo (30), se encontró que después de una corta instrucción, once endoscopistas con varios niveles de experiencia predijeron el resultado histológico con una precisión del $84 \%$. Hallazgos parecidos fueron encontrados por Neuman (33), con una precisión del $94,3 \%$ y una curva de aprendizaje que puede ser asimilada rápidamente. El uso rutinario del i-Scan permitiría a los examinadores menos hábiles alcanzar resultados comprables con endoscopistas experimentados.

\section{Posición de la ASGE respecto a la caracterización de pólipos colónicos y la cromoendoscopia virtual}

La American Society Gastrointestinal Endoscopy (ASGE) ha desarrollado una política de incorporación y preservación de las innovaciones endoscópicas (PIVI) (34) dirigidas en este caso en particular a la valoración en tiempo real de los pólipos diminutos $(<5 \mathrm{~mm})$. Recomienda dos estrategias: la primera, predecir-resecar y desechar los pólipos menores de $5 \mathrm{~mm}$ no localizados en el recto-sigmoides y que sean caracterizados en la cromoendoscopia virtual con alta confiabilidad; la segunda, predecir y no resecar los pólipos diminutos del recto-sigmoides menores de $5 \mathrm{~mm}$, caracterizados con alta confiabilidad en la cromoendoscopia digital de alta definición. Para utilizar estas estrategias es necesario enfatizar en los niveles de confidencia del diagnóstico óptico reduciendo la variación interobservador $(35,36)$. Una reciente revisión y metaanálisis de la ASGE, al igual que el estudio HISCOPE, plantean que los umbra-

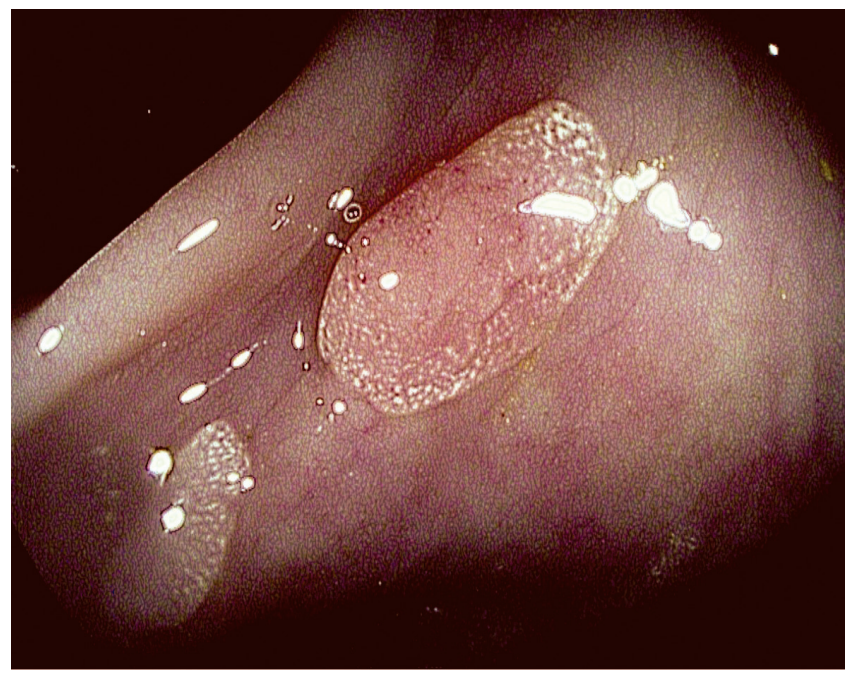

Figuras 1 y 2. Caracterización de pólipos sésiles OIs menores de $5 \mathrm{~mm}$, de superficies pálidas con criptas redondas de tamaño uniforme y vasos capilares aislados (no adenomatosos según clasificación ICE); observados con luz blanca de alta definición y filtro de cromoendoscopia virtual i-Scan 3 , respectivamente. 


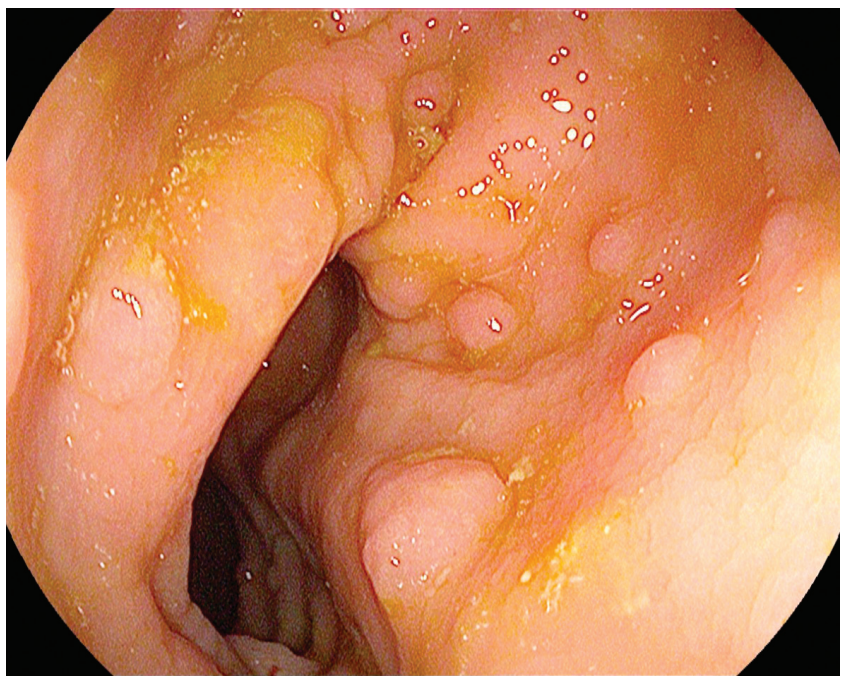

Figura 3. Varios pólipos diminutos en colon sigmoides observados con luz blanca de alta definición i-Scan, caracterizados según clasificación ICE como no adenomatosos.

les propuestos por PIVI pueden ser potencialmente alcanzados por el sistema i-Scan, pero es necesario el diseño de grandes estudios aleatorizados para comprobarlo $(37,38)$, además del entrenamiento y la acreditación que garanticen la calidad del diagnóstico óptico (39).

Sin embargo, es importante anotar que el sistema i-Scan colonoscopia es una herramienta complementaria a una buena técnica y a los indicadores de calidad en colonoscopia: intubación cecal, tiempo de retiro adecuado, porcentaje de detección de adenomas y la limpieza óptima del colon $(40,41)$.

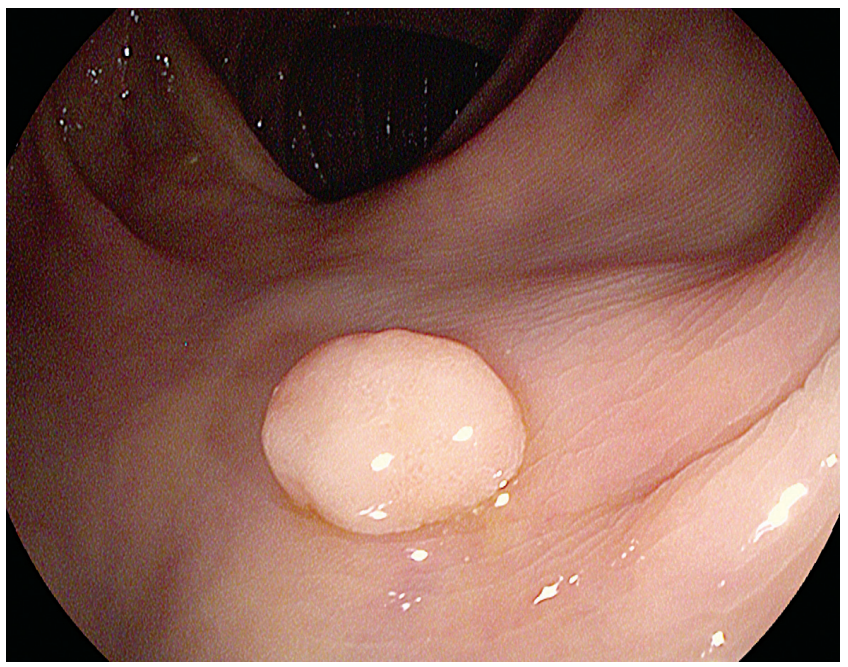

\section{Posición de la ESGE respecto a la caracterización de pólipos colónicos y la cromoendoscopia}

La Sociedad Europea de Endoscopia Gastrointestinal (ESGE) (42), en una guía reciente sobre el uso de las nuevas tecnologías en la detección y caracterización de las neoplasias del colon, recomienda:

- El uso rutinario de la endoscopia de alta magnificación con luz blanca para detectar neoplasias colorrectales en la población promedio.

- El uso rutinario de sistemas de alta definición y la pancromoendoscopia convencional o virtual (NBI, i-Scan) en pacientes conocidos o con sospecha de síndrome de Lynch.

- Que la cromoendoscopia convencional o virtual (NBI; FICE o i-Scan) debe ser estrictamente controlada en cuanto al diagnóstico en tiempo real de los pólipos menores o iguales de $5 \mathrm{~mm}$, para reemplazar el diagnóstico histopatológico. Además, debe fotodocumentarse $y$ realizarse por endoscopistas experimentados debidamente entrenados.

\section{ÁREAS DE INVESTIGACIÓN FUTURA}

Es necesario el consenso de los actuales sistemas de clasificación de las lesiones de la mucosa gastrointestinal encontradas con los endoscopios de alta definición para ser usados fácilmente en el escenario clínico. Además, debe estudiarse el valor de la cromoendoscopia digital comparado con la histología de los pólipos en relación con el PIVI planteado por la ASGE (43), para desarrollar módulos de entrenamiento certificados para su aplicación (44).

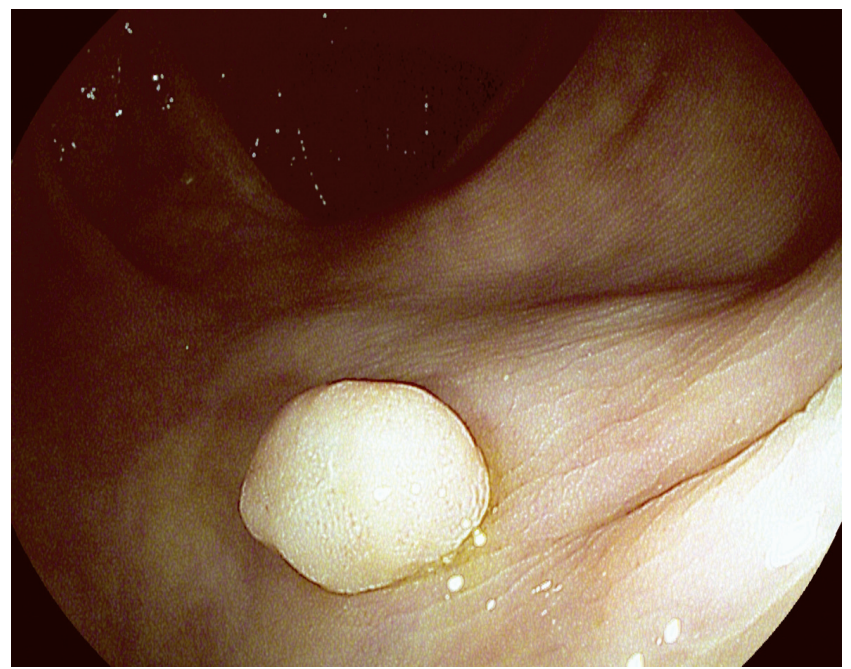

Figuras 4 y 5. Caracterización endoscópica de pólipo sésil menor de $5 \mathrm{~mm}$, patrón de criptas redondas de igual tamaño observadas con luz blanca de alta definición y luego con i-Scan 2. Clasificado según ICE no adenomatoso. 

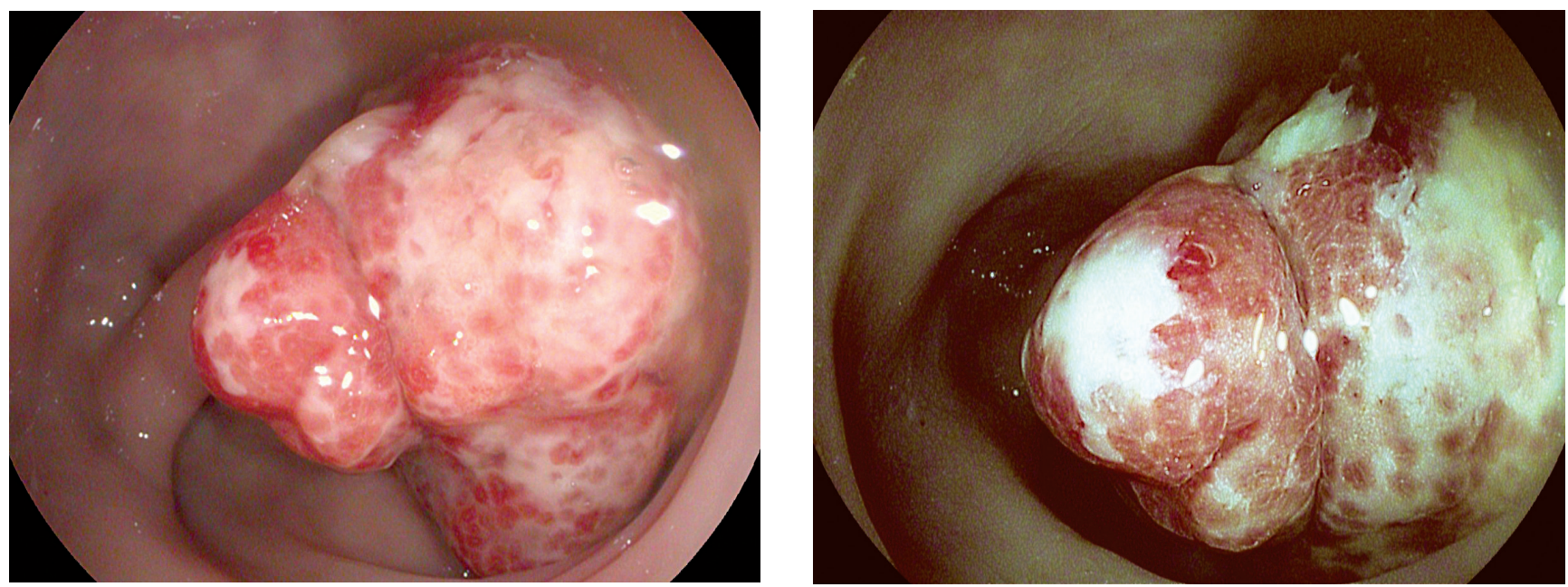

Figuras 6 y 7. Caracterización de pólipo pediculado de $0 I p$ de $15 \mathrm{~mm}$, de características adenomatoso con fosos ovalados y tubulares según clasificación ICE, observados con cromoendoscopia virtual i-Scan 1 y 2 , respectivamente.
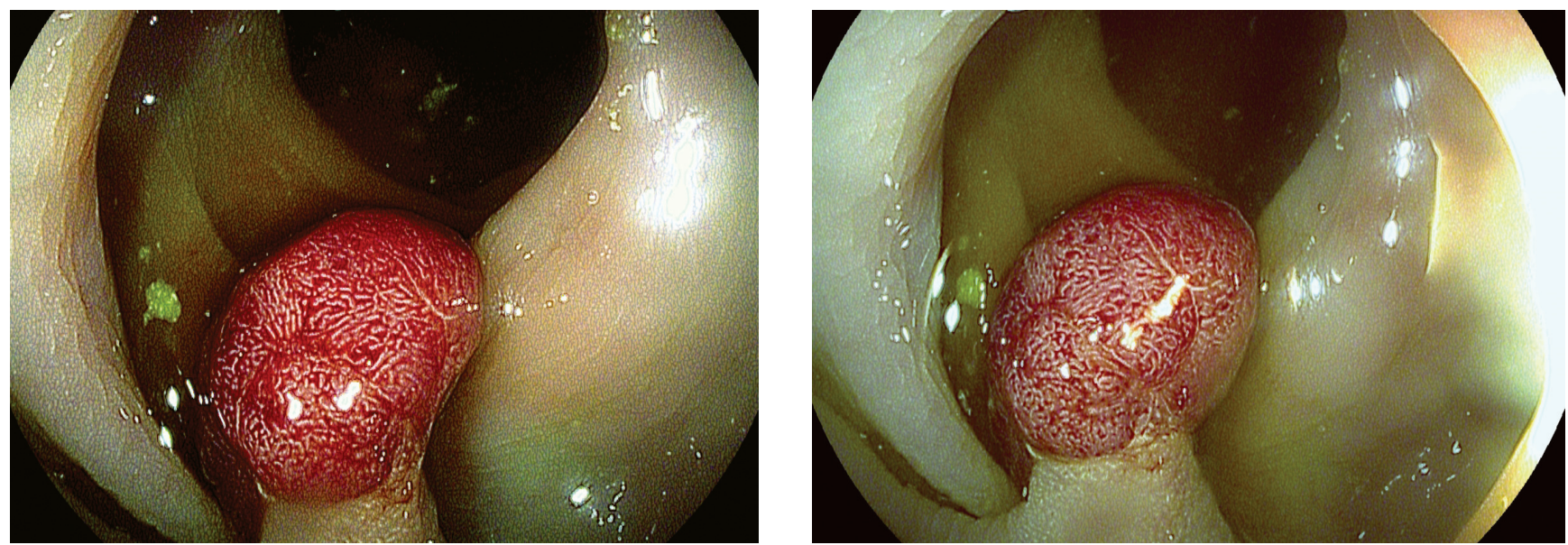

Figuras 8 y 9. Caracterización de pólipo pediculado 0Ip de $10 \mathrm{~mm}$, de color rojizo, observado con cromoendoscopia virtual i-Scan 2 y 3 , respectivamente. Adenomatoso según clasificación ICE.

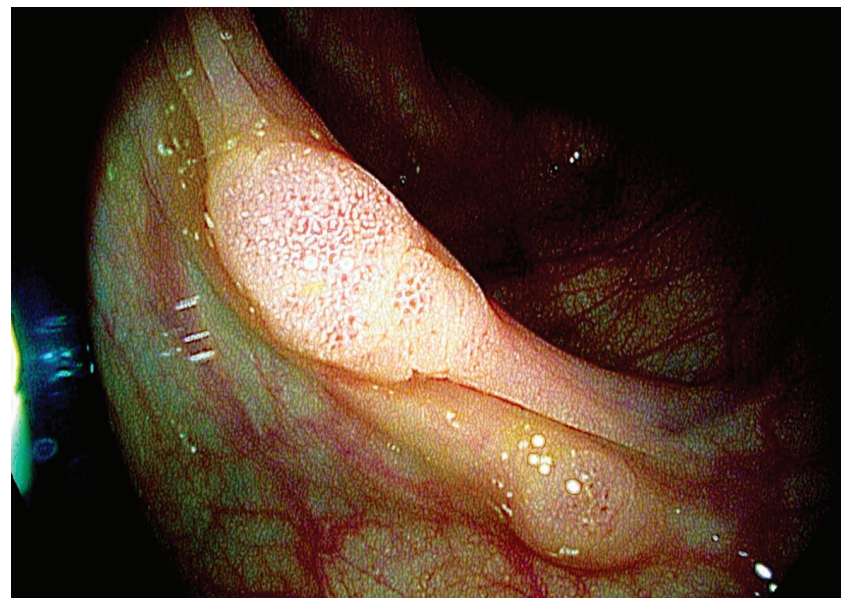

Figura 10. Caracterización de pólipo sésil menor de $5 \mathrm{~mm}$, OIs con criptas ovales y tubulares, patrón de vasos capilares dilatados, observado con cromoendoscopia virtual i-Scan 2, clasificación ICE adenomatoso.

\section{CONCLUSIONES}

El sistema i-Scan de alta definición puede ser considerado como una alternativa a los sistemas de cromoendoscopia virtual ya conocidos en la detección de neoplasias de colon.

Con la tecnología i-Scan, la predicción histológica de pólipos adenomatosos o hiperplásicos en tiempo real durante la colonoscopia es segura y su curva de aprendizaje puede ser adquirida rápidamente, lo cual podría implementar su uso rutinario y no ser exclusivo de centros de referencia.

\section{Conflicto de intereses}

El autor declara que no tiene conflicto de intereses. 


\section{REFERENCIAS}

1. Winawer SJ, Zauber AG, Ho MN, et al. Prevention of colorectal cancer by colonoscopy polypectomy. The National Polyp Study Workgroup. N Engl J Med. 1993;329:1977-81.

2. Winawer SJ, Zauber AG, O’Brien MJ, et al. Randomized comparison of surveillance intervals after colonoscopic removal of newly diagnosed adenomatous polyps. N Engl J Med. 1993;328:901-6.

3. Bressler B, Paszat LF, Chen Z, et al. Rates of new or missed colorectal cancers after colonoscopy and their risk factors: a population-based analysis. Gastroenterology. 2007;132:96-112.

4. Van Rijn JC, Eitsma JB, Stoker J, et al. Polyp miss rate determined by tandem colonoscopy: a systematic review. Am J Gastroenterol. 2006;101:343-50.

5. Rex DK, Cutler CS, Lemmel GT, et al. Colonoscopic miss rates of adenomas determined by back to back colonoscopies. Gastroenterology. 2007;132:96-102.

6. Devuni D, Vaziri H, Anderson J. Chromocolonoscopy. Gastroenterol Clin N Am. 2013;42:521-45.

7. Kudo S, Hirota S, Nakajima T, et al. Colorectal tumours and Pit Pattern. J Clin Pathol. 1994;47:880-5.

8. Tischendorf J, Wasmuth HE, Koch A, et al. Value of magnifying chromoendoscopy and narrow band imaging (NBI) in classifying colorectal polyps a prospective controlled study. Endoscopy. 2007;39:1092-6.

9. Huang Q, Fukami N, Kashida H, et al. Interobserver and Intraobserver consistency in the endoscopic assessment of colon pit patterns. Gastrointest Endosc. 2001;60:520-6.

10. Lopez- Cerón L, Sanabria E, Pellise M. Colonic polyps is it useful to characterize them with advance endoscopy? World J Gastroenterol. 2014;26:8449-57.

11. Neuman H, Fujishro M, Wilcox M, et al. Present and future perspectives of virtual chromoendoscopy with i-scan and optical enhancement technology. Dig Endosc. 2014;26(Suppl 1):43-51.

12. Neuman H, Nagel A, Buda A. Advance endoscopic imaging to improve adenoma detection. World J Gastrointest Endosc. 2015;7:224-9.

13. Reyes-Dorantes A. Nuevas técnicas de imagen (i-Scan, NBI, FICE). Rev Gastroenterol Mex. 2011;76:(Suppl 1):134-6.

14. Yeung TM, Mortesen NJ. Advances in endoscopic visualization of colorectal polyps. Colorectal Dis. 2011;13:352-9.

15. Galloro G. High technology imaging in digestive endoscopy. World J Gastrointest Endosc. 2012;16;4(2):22-7.

16. Masci E, Mangiavillano B, Costa C, et al. Interobserver agreement among endoscopists on evaluation of polypoid colorectal lesions visualized with Pentax i-Scan Technique. Dig Liver Dis. 2013;45:207-10.

17. Banks M, Haidry R, Butt M, et al. High resolution colonoscopy in a bowel cancer screening program improves polyp detection. World J Gastroenterol. 2011;17:4308-13.

18. Kwon RS, Adler DG, Chand B, et al. High-resolution and High-magnification endoscopes. Gastrointetinal Endosc. 2009;69:399-407.
19. Ventakaraman S, Ragunath K. Advance endoscopic imaging: a review of commercially aviable technologies. Clin Gastroenterol Hepatol. 2014;12:368-76.

20. Hancock S, Bowman E, Prabakaran J, et al. Use of i-scan endoscopic image enhancement technology and therapeutic endoscopy: a case series and review of the literature. Diagn Ther Endosc. 2012;2012:193570.

21. Kodashima S, Fujishiro M. Novel image-enhanced endoscopy with i-scan technology. World J Gastroenterol. 2010;16:1043-9.

22. Cho JH. Advanced imaging technology other than narrow band imaging. Clin Endosc. 2015:48:503-10.

23. Hoffman A, Sar F, Goetz M, et al. High definition colonoscopy combined with i-scan is superior in the detection of colorectal neoplasias compared with standard videocolonoscopy: a prospective randomized controlled trial. Endoscopy. 2010;47:827-33.

24. Hoffman A, Loth L, Rey JW, et al. High definition plus colonoscopy combined with i-scan tone enhancement vs. high definition colonoscopy for colorectal neoplasia: A randomized trial. Dig Liver Dis. 2014;46:991-6.

25. Bowman E, Pfau P, Mitra A, et al. High definition colonoscopy combined with i-SCAN imaging technology is superior in the detection of adenomas and advanced lessions compared to high definition colonoscopy alone. Diagn Ther Endosc. 2015;2015:167406.

26. Hong SN, Choe WH, Lee JH, et al. Prospective, randomized, back-to-back trial evaluating the usefulness of i-SCAN in screening colonoscopy. Gastrointest Endosc. 2012;75(5):1011-21.

27. Hoffman A, Kagel C, Goetz M, et al. Recognition and characterization of small colonic neoplasia with-definition colonoscopy using i-scan as precise chromoendoscopy. Dig Liver Dis. 2009;42;45-50.

28. Pigo F, Bertani H, Barbera C, et al. I-Scan high-definition white light endoscopy and colorectal polyps: prediction of histology interobserver and intraobserver agreement. Int J Colorectal Dis. 2013;28:399-406.

29. Lee Ch, Lee SH, Hwagbo Y. Narrow-band imaging versus I-Scan for the real time histological prediction of diminutive colonic polyps: a prospective comparative study by using the simple unified endoscopic classification. Gastrointest Endosc. 2011;74:603-9.

30. Hong SN, Choe WC, Lee JH, et al. Prospective, randomized, back-to-back trial evaluating the usefulness of i-SCAN screening colonoscopy. Gastrointest Endosc. 2012;75:1011-21.

31. Wanders L, East J, Uitentius S, et al. Diagnostic performance of narrowed a spectrum endoscopy autofluorescense imaging, and confocal laser endomicroscopy for optical diagnosis of colonic polyps: a meta-analysis. Lancet. 2013;14:1337-47.

32. Bouwens M, Ridder R, Masclee A, et al. Optical diagnosis of colorectal polyps using high-definition i-scan: an educational experience. World J Gastroenterol. 2013;19:4334-43.

33. Neuman H, Vieth M, Fry L, et al. Learning curve of virtual chromo endoscopy for the prediction of hyperplastic and 
adenomatous colorectal lesions: a prospective 2-center study. Gastrointest Endosc. 2013;78:115-20.

34. Rex DK, Kahi C, O'Brien M, et al. The American Society for Gastrointestinal Endoscopy PIVI (Preservation and Incorporation of Valuable Endoscopic Innovations) on realtime endoscopic assessment of the histology of diminute colorectal polyps. Gastrointest Endosc. 2011;73:419-22.

35. Kaltenbach T, Rex D, Wilson A, et al. Implementation of optical diagnosis for colorectal polyps: standardization of studies is needed. Clin Gastroenterol Hepatol. 2015;13:6-10.

36. Hassan C, Repici A, Zullo A, et al. New paradigms for colonoscopic management of diminutive colorectal polyps: predict, resect, and discard or do not resect? Clin Endosc. 2013;48:130-7.

37. Abu Dayyeh BK, Thosani N, Konda V, et al. ASGE Technology Committee systematic review and meta-analysis assessing the ASGE PIVI thresholds for adopting realtime endoscopic assessment of the histology of diminutive colorectal polyps. Gastrointest Endosc. 2015;81:502-16.

38. Basford P, Longcroft-Wheaton G, Higgins B, et al. Highdefinition endoscopy with i-Scan for evaluation of small colon polyps: the HisCOPE study. Gastrointest Endosc. 2014;79:111-8.

39. Wilson BA. Optical diagnosis of small colorectal polyps during colonoscopy: When to resect and during colonoscopy: When to resect and discard? Best Pract Res Clin Gastroenterol. 2015;29:639-49.

40. Gomez V, Wallace M. Advances in diagnostic and therapeutic colonoscopy. Curr Opin Gastroenterol. 2014;30:63-8.

41. Kiesslich R. Novel colonoscopic imaging techniques. Gastroenterol Hepatol. 2013;9:241-4.

42. Kaminski M, Hassan C, Bisschops R, et al. Advance imaging for detection and differentiation of colorectal neoplasia: European Society of Gastrointestinal Endoscopy (ESGE) Guideline. Endoscopy. 2014:46;435-49.

43. Bath Y, Abu Dayyeh B, Chauhan S, et al. High-definition and high-magnification endoscopes. Gastrointest Endosc. 2014;80:919-27.

44. Jang JY. The past, present, and future of image- enhanced. Endoscopy Clin Endosc. 2015;48:466-75. 